\title{
EXAMPLES OF NONTRIVIAL $H$-COBORDISMS
}

BY E. H. CONNELL

Communicated by R. H. Bing, August 17, 1967

Farrell and Hsiang have shown that $\exists$ an $h$-cobordism $W$ with ends $M$ and $M_{1}$ which are not homeomorphic [11]. Thus $W$ is not topologically trivial, i.e., is not homeomorphic to $M \times I$. The purpose of this note is to show that if $n \geqq 6$ and $M^{n-1}$ is the boundary of a regular neighborhood $X^{n}$ of a 2-complex, and $M^{n}$ is any p.l. $h$ cobordism which has $M$ as one end, then $W$ is homeomorphic to $M \times I$ iff the Whitehead torsion $\tau(W, M)=0$. Thus $W$ is topologically trivial iff it is p.l. trivial. A corollary is that for any finitely generated group $\pi$ with $\mathrm{Wh}(\pi) \neq 0, \exists$ an $h$-cobordism $W$ with $\pi=\pi_{1}(W)$ which is not topologically trivial.

Thus these results do not contain, nor are they contained in, the results of Farrell and Hsiang. Their approach is partly algebraic in that certain special facts about Whitehead groups are used. The approach here is geometric, and is based upon the study of "geometric groups" [7].

Notation. If $K$ is a finite connected complex, an abstract regular neighborhood $X^{n}$ of $K$ is a compact p.l. $n$-manifold containing $K$ as a subcomplex such that $X$ collapses to $K$. If $B$ is a metric space and $A \subset B$, the statement that $B \delta$-deforms to $A$ means $\exists h: B \times I$ $\rightarrow B$ with

$$
\begin{aligned}
& h(x, 0)=x, \quad x \in B, \\
& h(x, t)=x, \quad x \in A, t \in I, \\
& h(x, 1) \in A, \quad x \in B,
\end{aligned}
$$

and the diameter of $h(x, I)$ is less than $\delta$ for all $x \in B$. In other words, $A$ is a $\delta$-deformation retract of $B$.

The following theorem is the basis for this note, and is Corollary 3 of Theorem 2 of [7].

THEOREM 1. Hyp. $Y$ is a p.l. $n$-manifold and $L \subset Y$ is a connected subcomplex with dim $L \leqq 2$.

Conclusion: $\exists \delta>0$ such that if $V$ is any finite complex and $h: V \rightarrow Y$ is a topological embedding such that $h \mid h^{-1}(L): h^{-1}(L) \rightarrow L$ is p.l. onto and such that $h(V) \delta$-deforms to $L$, then $h^{-1} \mid L: L \rightarrow V$ is a simply homotopy equivalence. 
Theorem 2. Suppose $K$ is a finite, connected 2-complex and $X^{n}$ is an abstract regular neighborhood of $K, n \geqq 6$, and $Y$ is a p.l. manifold and $f: X \rightarrow Y$ is a homeomorphism onto. Then $f$ is a simple homotopy equivalence.

Proof. Since $n \geqq 6, f$ is isotopic $(\bmod \partial X)$ to a homeomorphism which, when restricted to $K$ is a p.l. embedding ([9] or [8]). Thus suppose that $f$ itself has this property, and let $L=f(K)$. Now $\tau(f)$ $=\tau(i)$ where $i: L \rightarrow Y$ is the inclusion. Let $\delta>0$ satisfying the conclusion of Theorem 1.

Since $X$ is a regular neighborhood of $K, \exists$ a p.l. embedding $g: X \rightarrow X$ such that

(1) $g$ is p.l. isotopic to the Id: $X \rightarrow X$.

(2) $g \mid K=$ Id: $K \rightarrow K$.

(3) $L$ is a $\delta$-deformation retract of $f g(X)$.

Now define a topological embedding $h: Y \rightarrow Y$ by $h=f g f^{-1}$. Note that

(1) $h$ is topologically isotopic to Id: $Y \rightarrow Y$,

(2) $h \mid L=$ Id: $L \rightarrow L$,

(3) $L$ is a $\delta$-deformation retract of $h(Y)$.

Now apply Theorem 1 with $V=Y$ and obtain $h^{-1} \mid L: L \rightarrow Y$ is a simple homotopy equivalence. Since $h^{-1} \mid L$ is the inclusion $L \rightarrow Y$, it follows that $\tau(f)=0$.

Now let $\pi$ be any finitely generated group. Construct a finite, connected 2-complex $K$ with $\pi_{1}(K)=\pi$, and an abstract regular neighborhood $X^{n}$ of $K$ with $n \geqq 6$. Now let $\alpha \in W h(\pi)$ and construct a p.l. $h$-cobordism $W$ between $\partial X$ and some other manifold, with $W \cap X$ $=\partial X$ and $\tau(W, \partial X)=\tau(W \cup X, X)=\alpha$. This is possible by [5].

Theorem 3. $W$ is homeomorphic to $\partial X \times I$ iff $\alpha=0$.

Proof. If $\alpha=0, W$ is p.l. homeomorphic to $\partial X \times I$ (p.l. Smale theory). Now suppose $W$ is homeomorphic to $\partial X \times I$. Let $Y=W \cup X$ and note that $\exists$ a homeomorphism $f: X \rightarrow Y$ which is homotopic to the inclusion $X \rightarrow Y$. Now $\alpha=\tau(Y, X)=\tau(f)$ and by Theorem 2, $\tau(f)=0$.

\section{REFERENCES}

1. J. H. C. Whitehead, Simplicial spaces, nuclei, and m-groups, Proc. London Math. Soc. 45 (1939), 243-327.

2. - Simple homotopy types, Amer. J. Math, 72 (1950), 1-57.

3. - Simple homotopy types, Lectures at Math. Institute, Oxford, 1952.

4. - Combinatorial homotopy. I, II, Bull. Amer. Math. Soc. 55 (1949), 213245, 453-496. 
5. J. Stallings, On infinite processes leading to differentiability in the complement of a point, Differential and Combinatorial Topology, Princeton Univ. Press, Princeton, N. J., 1965.

6. J. Milnor, Whitehead torsion, Bull. Amer. Math. Soc. 72 (1966), 358-426.

7. E. Connell and John Hollingsworth, Geometric groups and Whitehead torsion, (to appear).

8. H. Gluck, Embeddings in the trivial range, Ann. of Math. 81 (1965), 195-210.

9. T. Homma, On the imbedding of polyhedra in manifolds, Yokohama Math. J. 10 (1962), 5-10.

10. S. Smale, Generalized Poincare conjecture in dimensions greater than 4, Ann. of Math. 74 (1961), 391-406.

11. F. T. Farrell and W. C. Hsiang, H-cobordant manifolds are not necessarily homeomorphic, Bull. Amer. Math. Soc. 73 (1967), 741-744.

12. F. T. Farrell, The obstruction to fibering a manifold over a circle, Bull. Amer. Math. Soc. 73 (1967), 737-740.

RICE UNIVERSITY 